\title{
ENHANCED STAR FORMATION RATES IN \\ BINARY INTERACTING ELLIPTICAL GALAXIES
}

\author{
TAPAN K CHATTERJEE \\ Facultad de Ciencias, Fisico-Matematicas, \\ Universidad A Puebla, (A.P. 1316), Puebla, Mexico
}

We study the stellar orbits, as a function of the binary motion of two identical ellipticals, under initial conditions marginally sufficient for strong interaction. The stars were initially given circularly symmetric velocities. The tidal effects cause a redistribution of stellar orbits, resulting in crowding of stars in shells; the same attaining its maximum intensity slightly after a pericentric passage. As the galaxies recede, the structure disperses gradually by expanding; but is restored, intensified and forms at a shorter radial distance as the galaxies return for a subsequent approach in a shrinking orbit. We give the stellar positions, projected perpendicular to the orbital plane, shortly after the first $(t \approx 0.5)$ and second $(t \approx 6)$ pericentric passages in the figures; (time being given in dimensionless units corresponding to mass $=1$, radius $=1, G=4.50$ ). On the basis of the cooling gas inflow model, the gas will be compressed and shocked in these regions of enhanced stellar density, leading to bursts of star formation. The interval between the two successive starbursts is found to be of the same order as the trapping time needed by the galaxy to incorporate the gas ejected by stars in its cooling flow.
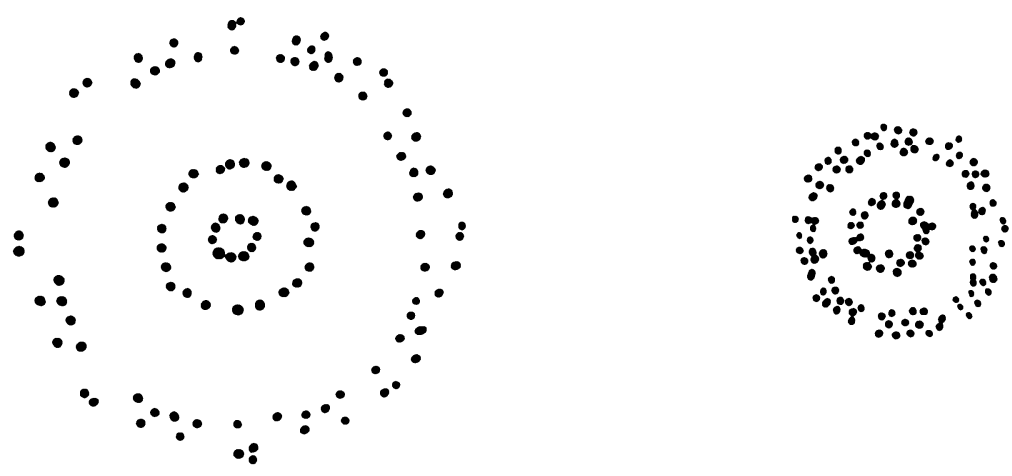

$+\approx 0.5$

$+\approx 6$ 